\title{
La nutrición como herramienta fundamental de salud pública
}

\author{
Nutrition as a fundamental public health tool
}

Oriana Rivera-Lozada ${ }^{1}$

oriana.rivera@uwiener.edu.pe

Artículo recibido: 25/02/2022

Artículo aceptado: 01/03/2022

Artículo publicado: 04/03/2022

Autor de correspondencia

Oriana Rivera-Lozada,

oriana.rivera@uwiener.edu.pe

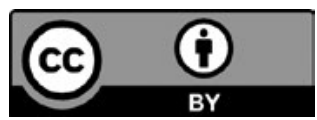

(CLa autora, 2022. Publicado por la Universidad Norbert Wiener (Lima, Perú)

Citar como: Rivera-Lozada O. La nutrición como herramienta fundamental de salud pública. Rev. Inv. UNW. 2022; 11(1): e0001.

doi: https://doi.org/10.37768/unw.rinv.11.01.e0001
La Organización Mundial de la Salud (OMS) define la nutrición como la ingesta de alimentos relacionada con la necesidad dietética del organismo. Por ello, una buena nutrición es el elemento básico para llevar una vida saludable $\left({ }^{1}\right)$. En los dos últimos años, la pandemia de COVID-19 ha originado una reacción de emergencia que obligó a una readaptación de los sistemas sanitarios $\left({ }^{2}\right)$ y confirmó que una nutrición adecuada permite una mejor respuesta en caso de contagio $\left({ }^{3}\right)$. Asimismo, el aprendizaje de nuevos tratamientos que pudieran hacer frente a un virus desconocido incluyó la atención nutricional como un componente básico para la recuperación de los pacientes $\left({ }^{2}\right)$.

Lo expuesto es una de las muchas razones por las que la Revista de Investigación de la Universidad Norbert Wiener ha considerado la preparación de un número especial dedicado a la nutrición, en el que se aborda tanto este tema como otras preocupaciones que los profesionales de la salud pública del mundo aún discuten.

La OMS estima que la anemia está presente en 1,24 billones de individuos en el mundo $\left({ }^{4}\right)$. Zoraida Arco aborda el problema como uno de los más persistentes en el mundo y que no ha podido ser desterrado, por lo que se pregunta en qué medida las prácticas de los padres corresponden con lo que se conoce sobre el tratamiento de la anemia. En ese mismo ámbito, Rosa Elena Cruz, Iván Carbajal, Saby Mauricio Alza y Michelle Lozada-Urbano evalúan si persistir en la educación alimentaria (preparación de recetas ricas en hierro, por ejemplo) es una estrategia válida para reducir la anemia en niños de 6 a 35 meses, y muestran como resultado una reducción del $93,7 \%$ al $23,9 \%$ en una intervención controlada. Beatriz Bonilla-Untiveros y Bárbara Rivero giran el foco hacia la prevalencia de la anemia en las mujeres embarazadas, una condición frecuente en el mundo (38,2\% de las gestantes) $\left({ }^{5}\right)$, y evalúan los tratamientos con hierro polimaltosado y sulfato ferroso, habituales en el Perú.

\footnotetext{
${ }^{1}$ Vicerrectorado de Investigación, Universidad Norbert Wiener. Lima, Perú.
} 
También en el ámbito de la salud pública se inscribe el trabajo de Jéssica León Garay, María Luisa Dextre y Michelle Lozada-Urbano, quienes examinan el contenido de azúcar, grasas saturadas y sodio en los productos industrializados que se destinan a lactantes y niños pequeños, y evidencian un relajamiento por parte de la autoridad sanitaria peruana respecto de la vigilancia en la advertencia publicitaria de los productos para alimentación complementaria.

Rodrigo Daga repasa los resultados de la Encuesta Demográfica y de Salud Familiar (Endes) 2017 y se enfoca en los resultados que describen la práctica de la lactancia materna exclusiva en el Perú, una estrategia de salud pública que busca garantizar una nutrición adecuada y disminuir la mortalidad y la morbilidad infantil $\left({ }^{6}\right)$.

Desde Argentina, Ornella Ghiglione y Andrea López abordan el estudio de los patrones alimentarios en niños con síndrome de Down en la ciudad de Posadas, y cómo estas prácticas originan la obesidad característica en un alto porcentaje de dicha población $\left({ }^{7}\right)$. Y Ana Clara Monteverde, Elisa Fernanda Naef, Laura Melina Peruzzo, María Consuelo Eckerdt y Carolina Clerici examinan los cambios producidos en la alimentación a partir del aislamiento social, preventivo y obligatorio por causa de la COVID-19 en personas adultas de Entre Ríos (Argentina), en el marco de la curricularización (trabajos de campo) que practica la Cátedra de Metodología de la Investigación de la carrera de Nutrición de la Universidad Nacional de Entre Ríos.

Edgardo Palma-Gutiérrez, Patricia Savino y Laura Joy hacen una revisión del abordaje nutricional que requieren los pacientes con cáncer, el que debería circunscribirse al proceso de atención nutricional (PAN), un método de cuatro fases que puede mejorar la calidad de atención para esta población y revertir su malnutrición.

José Narrea y Manuel Ramos revisan la evidencia sobre el uso del monohidrato de creatina, un suplemento relacionado con la nutrición deportiva y que podría tener grandes beneficios en el sector clínico, como en el caso de enfermedades neurodegenerativas y trastornos psiquiátricos, por lo que abogan para que se lleven a cabo investigaciones a mayor escala y diseñadas para humanos. También desde Argentina, Cecilia Cañulaf, Valeria Nepote y Liliana Ryan presentan los resultados de su proyecto de obtener harina a partir de la cascarilla desecada del fruto de la rosa mosqueta (usado frecuentemente para infusiones), valoran su aporte nutricional y evalúan la aceptación sensorial de galletas dulces elaboradas con esta harina, lo que revaloraría los cultivos de la Patagonia argentina. Finalmente, Diego Quico Cano postula la necesidad de aplicar cambios en la metodología de enseñanza del estudiante de nutrición, para educarlo en la importancia de la investigación.

Este número especial sobre nutrición, un aporte importante de la Revista de Investigación de la Universidad Norbert Wiener a la discusión de los temas abordados, no hubiese sido posible sin el valioso concurso de la nutricionista y magíster Catherine Bonilla, docente dela carrera de Nutrición Humana, y de su directora, la nutricionista y doctora en salud pública Saby Mauricio. Tenemos la firme convicción de continuar por esta ruta en futuras ediciones de la revista. 


\section{REFERENCIAS BIBLIOGRÁFICAS}

1. Gestarsalud. Día Mundial de la Nutrición: la alimentación se debe basar en nutrientes, no en calorías [Internet]. Colombia: Gestarsalud; 2021. Disponible en: https://gestarsalud.com/2021/05/28/ dia-mundial-de-la-nutricion-la-alimentacion-sedebe-basar-en-nutrientes-no-en-calorias/

2. Ballesteros M, Bretón I. Nutrición Clínica en tiempos de COVID-19. Endocrinol Diabetes Nutr. 2020; 67(7): 427-30. doi: 10.1016/j.endinu.2020.05.001

3. Universidad Peruana Los Andes. Conoce la importancia de la nutrición en pandemia [Internet]. Huancayo: UPLA; 2021. Disponible en: https://upla. edu.pe/conoce-la-importancia-de-la-nutricion-enpandemia/

4. Vos T, Abajobir AA, Abate KH, Abbafati C, Abbas KM, Abd-Allah F, Abera, SF. Global, regional, and national incidence, prevalence, and years lived with disability for 328 diseases and injuries for 195 countries, 1990-2016: a systematic analysis for the Global Burden of Disease Study 2016 [published correction appears in Lancet. 2017 Oct 28; 390 (10106): e38]. Lancet. 2017; 390(10100): 1211-59. doi: 10.1016/S0140-6736(17)32154-2
5. World Health Organization. The global prevalence of anaemia in 2011 [Internet]. 2015 [citado el 5 de mayo de 2021]. Disponible en: http://apps. who.int/iris/bitstream/10665/66914/1/WHO_ NHD_01.3.pdf?ua=1

6. Lutter CK, Morrow AL. Protection, promotion, and support and global trends in breastfeeding. Adv Nutr. 2013; 4(2): 213-9.

7. Roizen NJ, Patterson D. Down's syndrome. Lancet. 2003; 361(9365): 1281-9. 